\title{
TRANSPORT PROPERTIES OF PARTIALLY IONIZED ATOMIC GASES WITH ELECTRONIC EXCITATION
}

\section{A. Istomin and E. V. Kustova}

Department of Mathematics and Mechanics, Saint Petersburg State University 28 Universitetskiy pr., St. Petersburg 198504, Russia

\begin{abstract}
Chemically nonequilibrium ionized gas mixture flows of $\left(\mathrm{N}, \mathrm{N}^{+}, e^{-}\right)$and $\left(\mathrm{O}, \mathrm{O}^{+}, e^{-}\right)$with electronic excitation of neutral component are studied. The one-temperature model of transport properties taking into account electronic states of neutral atoms is developed. The transport coefficients of thermal and electrical conductivity, diffusion and thermal diffusion, shear and bulk viscosity are calculated in the temperature range $500-50,000 \mathrm{~K}$ for various mixture compositions. Contribution of the electronic degrees of freedom to the transport properties is found to be important.
\end{abstract}

\section{INTRODUCTION}

Transport properties of gases with internal degrees of freedom and chemical reactions are widely discussed in the literature [1-4]. However, in the majority of studies, only translational, rotational, and vibrational degrees of freedom are taken into account in the transport algorithms. In [5], it was shown that electronic excitation influences significantly the internal specific heats and thus may affect also the transport coefficients. When electronic degrees of freedom are neglected, atomic species are considered as structure-less particles possessing only translational energy; therefore, neither internal heat conductivity nor bulk viscosity appear in the expressions for the heat flux and mean normal stress. In high-temperature flows, such assumptions may lead to inaccurate predictions of flow parameters.

The transport processes in the electronically excited hydrogen plasma $\left(\mathrm{H}, \mathrm{H}^{+}\right.$, $\left.e^{-}\right)$near chemical equilibrium were extensively studied in $[6,7]$ except the effect of bulk viscosity. In the authors' recent papers [8, 9], nonequilibrium neutral mixtures $\mathrm{N}_{2} / \mathrm{N}$ and $\mathrm{O}_{2} / \mathrm{O}$ with electronically excited atoms and molecules were 
considered. In both weak and strong nonequilibrium cases, for the temperature higher than $10,000 \mathrm{~K}$, it was found that the coefficient of electronic heat conductivity is comparable and even exceeds that of the translational degrees of freedom.

In the present paper, the theoretical model proposed in $[8,9]$ is generalized to the case of high-temperature chemically nonequilibrium gas mixtures containing electrons, ions, and electronically excited atoms. The $\left(\mathrm{N}, \mathrm{N}^{+}, e^{-}\right)$ and $\left(\mathrm{O}, \mathrm{O}^{+}, e^{-}\right)$mixtures are considered. Using the modified Chapman-Enskog method [4], the closed set of governing equations in the zero- and first-order approximations is derived and the expressions for the diffusion velocities, electrical current density, heat flux, and stress tensor are obtained. The algorithm for the calculation of transport coefficients taking into account slow convergence of the Sonine polynomials is developed. The new effects of bulk viscosity and internal thermal conductivity in atomic species are discussed, and the conditions when the electronic states should be taken into account for the accurate evaluation of transport properties are specified.

\section{MACROSCOPIC PARAMETERS AND GOVERNING EQUATIONS}

In the current paper, a one-temperature chemically nonequilibrium flow of gas mixtures $\left(\mathrm{N}, \mathrm{N}^{+}, e^{-}\right)$and $\left(\mathrm{O}, \mathrm{O}^{+}, e^{-}\right)$is considered. For gas mixtures, it is assumed that the following relation between characteristic times is held:

$$
\tau_{\text {tr }}<\tau_{\text {el }} \ll \tau_{\text {ioniz }} \sim \theta .
$$

Here, $\tau_{\mathrm{tr}}, \tau_{\mathrm{el}}$, and $\tau_{\text {ioniz }}$ are the characteristic times for the elastic collisions, excitation of electronic degrees of freedom, and ionization; $\theta$ is the mean time of the gasdynamic parameters variation. For this relation, ionization can be considered on the basis of the maintaining Maxwell-Boltzmann distributions over the velocity and internal energy.

In the present study, the electronically excited states of atomic species are taken into account. Thus, the internal energy of atoms is modeled as $[8] \varepsilon_{c, l}^{\text {at }}$ $=\varepsilon_{c, \mathrm{el}}(l)$ where $\varepsilon_{c, \text { el }}(l)$ is the energy of the $l$ th electronic state of the chemical species $c$. The electronic states of atoms are provided in [5]. The ionized atoms and electrons are treated as unstructured particles.

The closed set of governing equations for the macroscopic parameters taking into account electronic degrees of freedom of atoms is derived from the kinetic equations for the distribution function $f_{\mathrm{cl}}(\mathbf{r}, \mathbf{u}, t)$ ( $\mathbf{r}$ is the coordinate, $\mathbf{u}$ is the particle velocity, and $t$ is the time) using the generalized Chapman-Enskog method $[4,8]$. In the absence of external forces and electromagnetic fields, the governing equations have the following form: 


$$
\begin{gathered}
\frac{d n_{c}}{d t}+n_{c} \nabla \cdot \mathbf{v}+\nabla \cdot\left(n_{c} \mathbf{V}_{c}\right)=R_{c}^{\text {ioniz }}, c=1,2,3 \\
\rho \frac{d \mathbf{v}}{d t}+\nabla \boldsymbol{P}=0 ; \\
\rho \frac{d U}{d t}+\nabla \cdot \mathbf{q}+\boldsymbol{P}: \nabla \mathbf{v}=0 .
\end{gathered}
$$

Here, $n_{c}$ is the number density of species $c ; \mathbf{v}$ is the gas velocity; $U$ is the total specific energy including electronic degrees of freedom; $\mathbf{V}_{c}$ is the diffusion velocity; $R_{c}^{\text {ioniz }}$ is the production term due to ionization; $\rho$ is the mixture density; $\boldsymbol{P}$ is the pressure tensor; and $\mathbf{q}$ is the heat flux.

\section{ZERO- AND FIRST-ORDER APPROACHES}

In the zero-order approximation of the modified Chapman-Enskog method, the distribution functions represent the local equilibrium Maxwell-Boltzmann distributions of atoms over the velocity and internal energy, and nonequilibrium distribution over chemical species:

$$
f_{\mathrm{cl}}^{(0)}=\left(\frac{m_{c}}{2 \pi k T}\right)^{3 / 2} \frac{n_{c} s_{c l}}{Z_{c}^{\text {int }}(T)} \exp \left(-\frac{m_{c} c_{c}^{2}}{2 k T}-\frac{\varepsilon_{l}^{c}}{k T}\right)
$$

where $m_{c}$ is the mass of a particle of the $c$ th species; $T$ is the temperature; $\mathbf{c}_{c}$ $=\mathbf{u}_{c}-\mathbf{v}$ is the peculiar velocity; $k$ is the Boltzmann constant; $s_{\mathrm{cl}}$ is the statistical weight for the internal state $l$; and $Z_{c}^{\text {int }}(T)$ is the equilibrium internal partition function:

$$
Z_{c}^{\mathrm{int}}(T)=\sum_{l} s_{\mathrm{cl}} \exp \left(-\frac{\varepsilon_{l}^{c}}{k T}\right) .
$$

A substantial difference from the models developed earlier is that for atoms, not only the Maxwell velocity distribution is obtained, but also the Boltzmann distribution over the electronic energy levels. For ionized atoms and electrons, the distribution function represents the Maxwell velocity distribution.

In the first-order approximation of the modified Chapman-Enskog method, the distribution function is obtained in the following form:

$$
=f_{\mathrm{cl}}^{(0)}\left(-\frac{1}{n} \mathbf{A}_{\mathrm{cl}}^{(1)} \cdot \nabla \ln T-\frac{1}{n} \sum_{d} \mathbf{D}_{\mathrm{cl}}^{d} \cdot \mathbf{d}_{d}-\frac{1}{n} \boldsymbol{B}_{\mathrm{cl}}: \nabla \mathbf{v}-\frac{1}{n} F_{\mathrm{cl}} \nabla \cdot \mathbf{v}-\frac{1}{n} G_{\mathrm{cl}}\right) .
$$

The first-order correction depends on the gradients of all macroscopic parameters and contains the unknown functions $\mathbf{A}_{\mathrm{cl}}, \mathbf{D}_{\mathrm{cl}}, \boldsymbol{B}_{\mathrm{cl}}, F_{\mathrm{cl}}$, and $G_{\mathrm{cl}}$ which are found from the linear integral equations similar to those derived in [4]. 
The expressions for the pressure tensor, diffusion velocity, energy flux, and electrical current flux in a viscous conductive flow are derived using the modified Chapman-Enskog method procedure:

$$
\begin{aligned}
\boldsymbol{P} & =\left(p-p_{\mathrm{rel}}\right) \boldsymbol{I}-2 \eta \boldsymbol{S}-\zeta \nabla \cdot \mathbf{v} I \\
\mathbf{V}_{c} & =-\sum_{d} D_{c d} \mathbf{d}_{c}-D_{T c} \nabla \ln T ; \\
\mathbf{q} & =-\lambda^{\prime} \nabla T-p \sum_{c} D_{T_{c}} \mathbf{d}_{c}+\sum_{c} \rho_{c} h_{c} \mathbf{V}_{c} ; \\
\mathbf{J} & =\sum_{c} n_{c} e_{c} \mathbf{V}_{c}=-\sum_{c d} \sigma_{c}^{d} \mathbf{d}_{d}^{\prime}-\sum_{c} \phi_{c} \nabla T .
\end{aligned}
$$

Here, $\boldsymbol{S}$ is the deformation rate tensor; $\boldsymbol{I}$ is the unity tensor; $\mathbf{d}_{c}$ is the diffusive driving force; $h_{c}$ is the specific enthalpy of species $c$; and $e_{c}$ is the charge of species $c$; and $\mathbf{d}_{c}^{\prime}$ is connected with diffusive driving force $\mathbf{d}_{c}$ in the following form:

$$
\mathbf{d}_{c}^{\prime}=\frac{p}{\rho_{c}}\left(\frac{e_{c}}{m_{c}}-\sum_{i} \frac{n_{i} e_{i}}{\rho}\right)^{-1} \mathbf{d}_{c} .
$$

In Eqs. (1)-(4), $\eta$ and $\zeta$ are the shear and bulk viscosity coefficients; $p_{\text {rel }}$ is the relaxation pressure; $D_{c d}$ and $D_{T c}$ are the diffusion and thermal diffusion coefficients; $\lambda^{\prime}=\lambda_{\mathrm{tr}}+\lambda_{\text {int }}$ is the partial thermal conductivity coefficient including contributions of the translational and internal degrees of freedom; and $\sigma_{c}^{d}$ and $\phi_{c}$ are the electrical conductivity and partial thermal-electric coefficients, respectively.

Note that the normal mean stress in this case includes the terms $\zeta \nabla \cdot \mathbf{v}$ and $p_{\text {rel }}$ associated, respectively, with the flow compressibility and the contribution of nonequilibrium ionization reactions.

\section{TRANSPORT COEFFICIENTS}

In order to calculate the transport coefficients, the unknown functions $\mathbf{A}_{\mathrm{cl}}, \mathbf{D}_{\mathrm{cl}}$, $\boldsymbol{B}_{\mathrm{cl}}, F_{\mathrm{cl}}$, and $G_{\mathrm{cl}}$ are expanded into the series of the Sonine polynomials in the reduced peculiar velocity $S_{\nu}^{(r)}$ and Waldmann-Trübenbacher polynomials in the internal energy $P_{l}^{(p)}[4]$. Note that in the case of ionized gases, due to the slow convergence of Sonine polynomials, it is not sufficient to keep in the expansions only the first nonvanishing terms. In the present study, the terms $r=p=0$; $r=1, p=0 ; r=2, p=0$; and $r=0, p=1$ are retained [2]. The transport coefficients are expressed in terms of the expansion coefficients: 


$$
\begin{gathered}
\lambda^{\prime}=\sum_{c} \frac{5}{4} k \frac{n_{c}}{n} a_{c, 10}+\sum_{c} \frac{m_{c}}{2} \frac{n_{c}}{n} c_{\mathrm{int}, c}, a_{c, 01} ; \\
D_{c d}=\frac{1}{2 n} d_{c, 0}^{d} ; \quad D_{T c}=-\frac{1}{2 n} a_{c, 00} ; \\
\eta=\frac{k T}{2} \sum_{c} \frac{n_{c}}{n} b_{c, 0} ; \quad \zeta=-k T \sum_{c} \frac{n_{c}}{n} f_{c, 10} ; \\
p_{\text {rel }}=k T \sum_{c} \frac{n_{c}}{n} g_{c, 10} ;
\end{gathered}
$$

and $c_{\text {int, } c}$ is the internal specific heat at constant volume related to the electronic degrees of freedom. On the basis of calculated diffusion coefficients $D_{c d}$, it is possible to calculate the electrical conductivity:

$$
\sigma_{c}^{d}=\frac{n_{c} e_{c} \rho_{c}}{p}\left(\frac{e_{c}}{m_{c}}-\sum_{i} \frac{n_{i} e_{i}}{\rho}\right) D_{c d} .
$$

The present authors use the standard procedure of the Chapman-Enskog method [4] and show that the integral equations for the unknown functions are reduced to the transport linear systems of algebraic equations involving the bracket integrals as coefficients. The bracket integrals are simplified applying the commonly used assumptions proposed by Mason and Monchick [1]. The transport coefficients are then calculated numerically as the solutions of the transport linear systems using the Gauss method [4].

Finally, all transport coefficients are expressed in terms of the collision integrals $\Omega_{c d}^{(l, r)}$ and integrals $\beta$ depending on the energy variation $\Delta \epsilon$ in inelastic collisions. The integrals $\Omega_{c d}^{(l, r)}$ are calculated using the data from [10] for all types of collisions: neutral-neutral, neutral-charged, and charged-charged interactions. For the transitions between electronic states, there is no accurate data on the relaxation times, which is necessary for calculating integrals $\beta$. The authors propose to approximate the corresponding integral using the deactivation rate constants for the transition from the first to the ground electronic state [9]. The deactivation rate constants $k_{10}$ are taken from $[11,12]: k_{10 \mathrm{~N}}=10^{-19} \mathrm{~m}^{3} / \mathrm{s}$ and $k_{100}=8 \cdot 10^{-18} \mathrm{~m}^{3} / \mathrm{s}$.

\section{RESULTS AND DISCUSSION}

The transport coefficients were calculated for the $\left(\mathrm{N}, \mathrm{N}^{+}, e^{-}\right)$and $\left(\mathrm{O}, \mathrm{O}^{+}, e^{-}\right)$ mixtures in the temperature range $500-50,000 \mathrm{~K}$ using the approach developed above. The results show that electronic excitation has a significant effect on the thermal conductivity coefficient. 


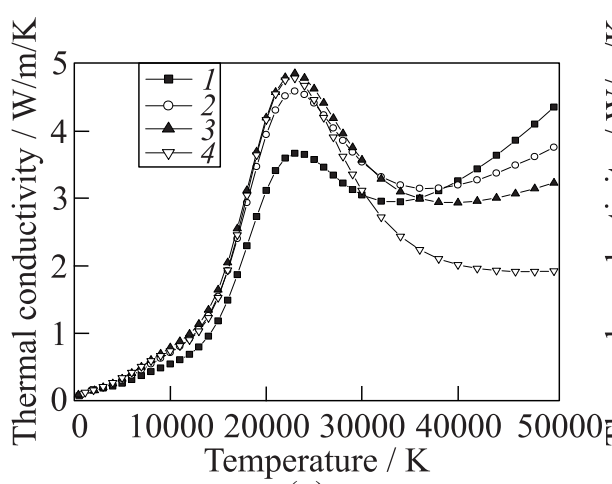

(a)

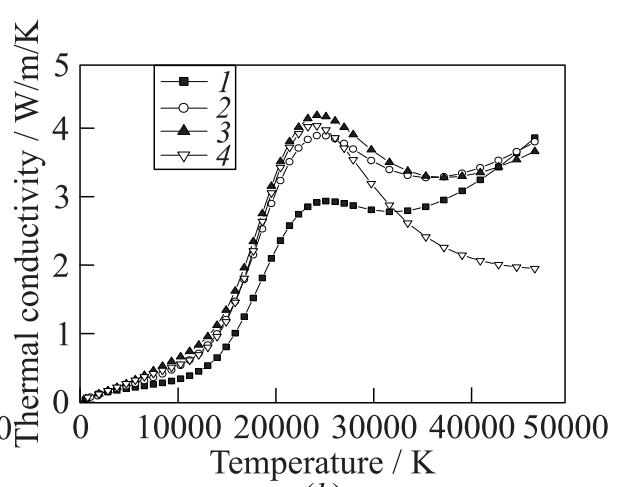

(b)

Figure 1 Thermal conductivity coefficient as a function of temperature for $\left(\mathrm{N}, \mathrm{N}^{+}, e^{-}\right)(a)$ and $\left(\mathrm{O}, \mathrm{O}^{+}, e^{-}\right)(b)$ mixtures: $1-x_{\text {atom }} / x_{\text {ion }} / x_{e}=0.90 / 0.05 / 0.05$; $2-0.96 / 0.02 / 0.02 ; 3-0.90 / 0.01 / 0.01 ;$ and $4-x_{\text {atom }} / x_{\text {ion }} / x_{e}=1 / 0 / 0$

In Fig. 1, the total thermal conductivity $\lambda^{\prime}$ as a function of $T$ is presented for four cases corresponding to different mixture compositions: $x_{\text {atom }}=0.9, x_{\text {ion }}$ $=x_{e}=0.05 ; x_{\text {atom }}=0.96, x_{\text {ion }}=x_{e}=0.02 ; x_{\text {atom }}=0.98, x_{\text {ion }}=x_{e}=0.01 ;$ and $x_{\text {atom }}=1, x_{\text {ion }}=x_{e}=0\left(x_{\text {atom }}, x_{\text {ion }}\right.$, and $x_{e}$ are the molar fractions of atoms, ions, and electrons). For a pure atomic gas, the thermal conductivity coefficient has a strong maximum in the temperature range $T=22,000-26,000 \mathrm{~K}$. The thermal conductivity coefficient $\lambda^{\prime}$ of mixtures also increases in this temperature interval; however, it remains lower than that for atoms, except for 98\%/1\%/1\% composition. Thus, one can conclude that the most significant contribution to the total thermal conductivity of $\left(\mathrm{N}, \mathrm{N}^{+}, e^{-}\right)$and $\left(\mathrm{O}, \mathrm{O}^{+}, e^{-}\right)$mixtures in the temperature range $T=22,000-26,000 \mathrm{~K}$ is given by electronic excitation of atomic components. In the temperature range $T=30,000-50,000 \mathrm{~K}$, the contribution of electrons to thermal conductivity becomes more important. This is explained by the fact that electrons are the light and fast particles, and with the temperature rise, their role in the heat transfer increases.

In Fig. 2, the contribution of the electronic states to the thermal conductivity coefficient $\lambda^{\prime}$ of atomic species and various mixtures of nitrogen and oxygen plasmas is shown. For such conditions, the coefficient of internal thermal conductivity $\lambda_{\text {int }}$ in the temperature range $18,000-24,000 \mathrm{~K}$ is approximately 4 times larger than the corresponding translational thermal conductivity coefficient. Thus, we can conclude that the effect of atomic electronic states on the thermal conductivity coefficients is significant.

Figure 3 presents the coefficient of thermal conductivity $\lambda^{\prime}$ calculated as a function of $x_{\text {atom }}$ at fixed temperatures: in the mixture $\left(\mathrm{N}, \mathrm{N}^{+}, e^{-}\right), T$ $=22,000 \mathrm{~K}$, and in the mixture $\left(\mathrm{O}, \mathrm{O}^{+}, e^{-}\right), T=24,000 \mathrm{~K}$. Fixed values of 


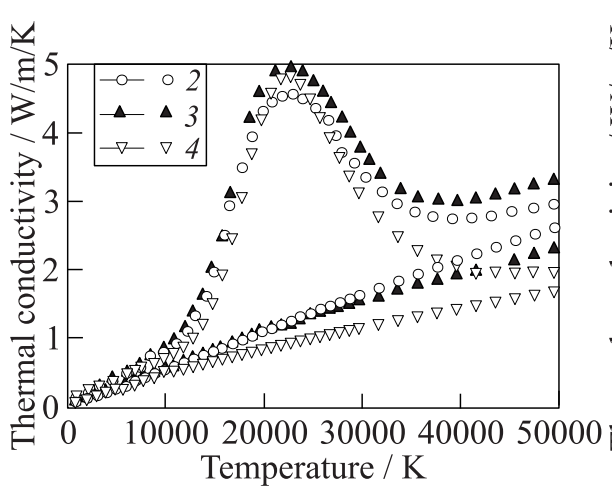

(a)

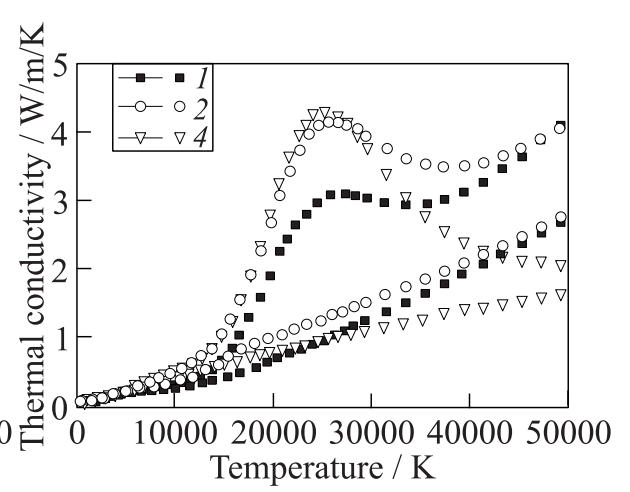

(b)

Figure 2 Total and translational thermal conductivity coefficient as a function of temperature for $\left(\mathrm{N}, \mathrm{N}^{+}, e^{-}\right)(a)$ and $\left(\mathrm{O}, \mathrm{O}^{+}, e^{-}\right)(b)$ mixtures. Curves with symbols correspond to atoms with electronic excitation whereas symbols designate the results obtained neglecting electronic states: $1-x_{\text {atom }} / x_{\text {ion }} / x_{e}=0.90 / 0.05 / 0.05$; $2-0.96 / 0.02 / 0.02 ; 3-0.90 / 0.01 / 0.01 ;$ and $4-x_{\text {atom }} / x_{\text {ion }} / x_{e}=1 / 0 / 0$

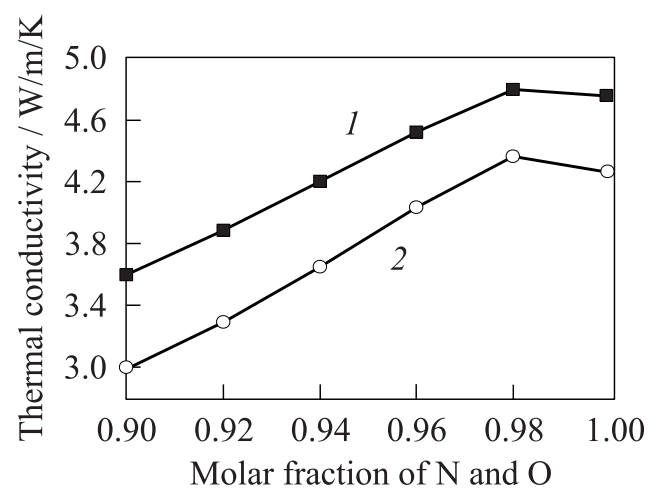

Figure 3 Thermal conductivity coefficient $\lambda^{\prime}$ as a function of $n_{\text {atom }}: 1-\mathrm{N} / \mathrm{N}^{+} / e^{-}$, $T=22,000 \mathrm{~K}$, and $2-\mathrm{O} / \mathrm{O}^{+} / e^{-}, T=24,000 \mathrm{~K}$

temperature correspond to the maxima of the atomic specific heats. The thermal conductivity coefficients have a maximum at 98\%/1\%/1\% mixture composition. But other compositions increase significantly with the rise of atomic species concentration. This confirms the authors' conclusion that the most important contribution to the thermal conductivity coefficients in the temperature range $T=15,000-35,000 \mathrm{~K}$ is given by atoms, and the main role belongs to the atomic electronic states. 


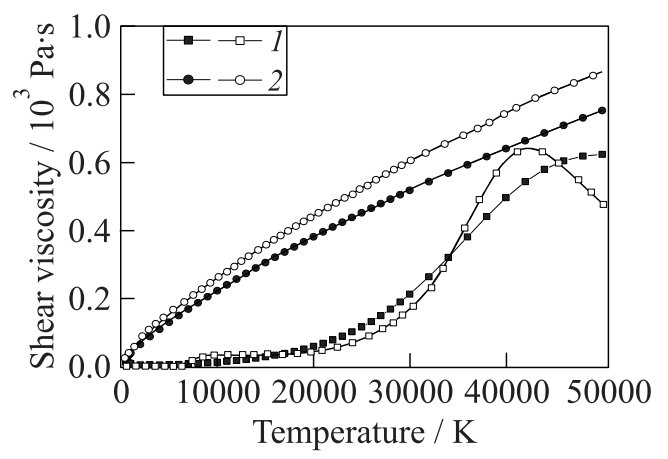

Figure 4 Shear viscosity coefficient $\eta$ as a function of $T(a): 1-x_{\text {atom }} / x_{\text {ion }} / x_{e}$ $=0.96 / 0.02 / 0.02 ;$ and $2-x_{\text {atom }} / x_{\text {ion }} / x_{e}=1 / 0 / 0$. Filled symbols refer to $\mathrm{N}$, and empty to $\mathrm{O}$

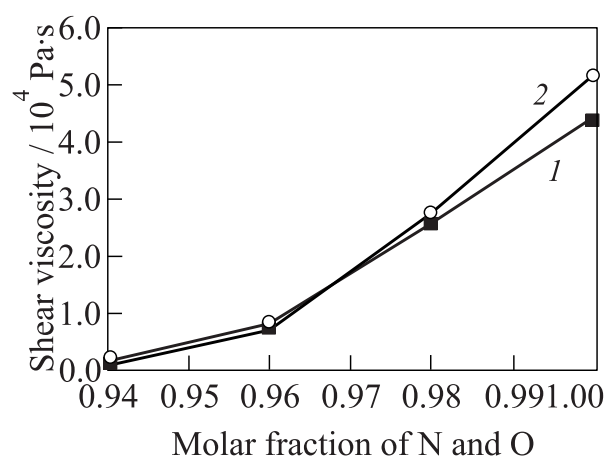

Figure 5 Shear viscosity coefficient $\eta$ as a function of $n_{\text {atom }}: 1-\mathrm{N} / \mathrm{N}^{+} / e^{-}, T$ $=22,000 \mathrm{~K} ;$ and and $2-\mathrm{O} / \mathrm{O}^{+} / e^{-}, T=24,000 \mathrm{~K}$

The coefficient of shear viscosity $\eta$ for the $\left(\mathrm{N}, \mathrm{N}^{+}, e^{-}\right)$and $\left(\mathrm{O}, \mathrm{O}^{+}, e^{-}\right)$mixtures is given in Figs. 4 and 5 as a function of $T$ and $x_{\text {atom. The shear viscosity }}$ for pure gases increases with temperature. For ionized mixtures, in the temperature range $T=40,000-50,000 \mathrm{~K}$, the contribution of electrons to shear viscosity becomes more important. For this temperature range, the shear viscosity coefficient slowly decreases.

Figures 6 and 7 present the coefficient of bulk viscosity $\zeta$ as a function of $T$ and $x_{\text {atom. }}$. With the rise of $T$, the bulk viscosity coefficient behaves nonmonotonically; for atomic oxygen, the bulk viscosity coefficient remains lower than that for atomic nitrogen. This is explained by the fact that the deactivation rate constants of atomic oxygen is about two orders of magnitude larger than that for 


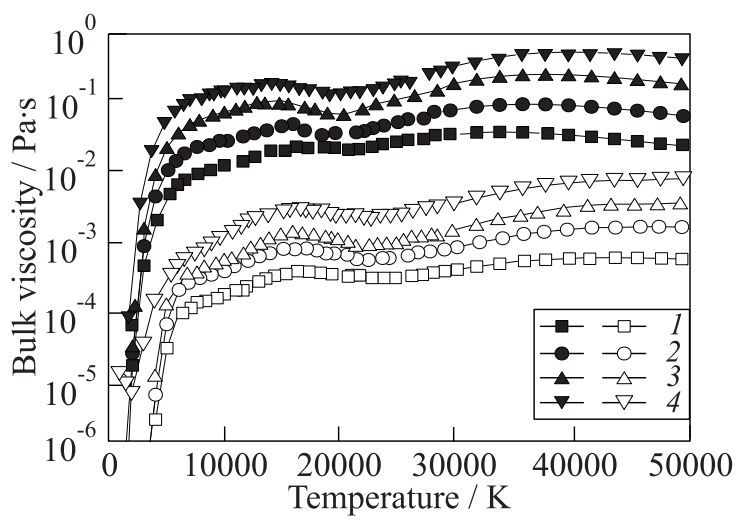

Figure 6 Bulk viscosity coefficient $\zeta$ as a functions of $T$ : $1-x_{\text {atom }} / x_{\text {ion }} / x_{e}$ $=0.90 / 0.05 / 0.05 ; 2-0.96 / 0.02 / 0.02 ; 3-0.98 / 0.01 / 0.01 ;$ and $4-x_{\text {atom }} / x_{\text {ion }} / x_{e}$ $=1 / 0 / 0$. Filled symbols refer to $\mathrm{N}$, and empty to $\mathrm{O}$

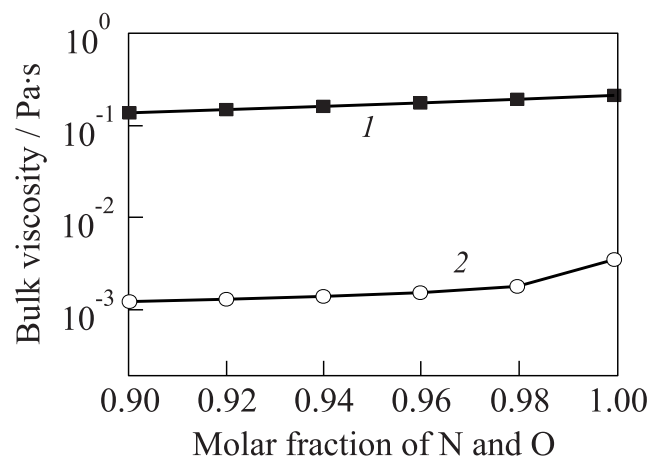

Figure 7 Bulk viscosity coefficient $\zeta$ as a functions of $n_{\text {atom }}: 1-\mathrm{N} / \mathrm{N}^{+} / e^{-}, T$ $=22,000 \mathrm{~K}$; and $2-\mathrm{O} / \mathrm{O}^{+} / e^{-}, T=24,000 \mathrm{~K}$

atomic nitrogen. For $\left(\mathrm{N}, \mathrm{N}^{+}, e^{-}\right)$and $\left(\mathrm{O}, \mathrm{O}^{+}, e^{-}\right)$mixtures, the bulk viscosity increases with the rise of the atomic molar fraction in the mixture.

The coefficient of electrical conductivity $\sigma_{c}^{d}$ as a function of $T$ for $\left(\mathrm{N}, \mathrm{N}^{+}, e^{-}\right)$ and $\left(\mathrm{O}, \mathrm{O}^{+}, e^{-}\right)$mixtures for constant mixture composition is given in Fig. 8 .

Analyzing the results, one can conclude that electronic excitation has a strong influence on thermal conductivity and bulk viscosity coefficients. On the other hand, its contribution to the remaining transport coefficients: shear viscosity, diffusion, and electrical conductivity is negligible. In the temperature ranges $T=500-5000 \mathrm{~K}$ for nitrogen and $T=500-7000 \mathrm{~K}$ for oxygen, the contribution of electronic excitation to the thermal conductivity coefficient is weak $(<7 \%)$. 


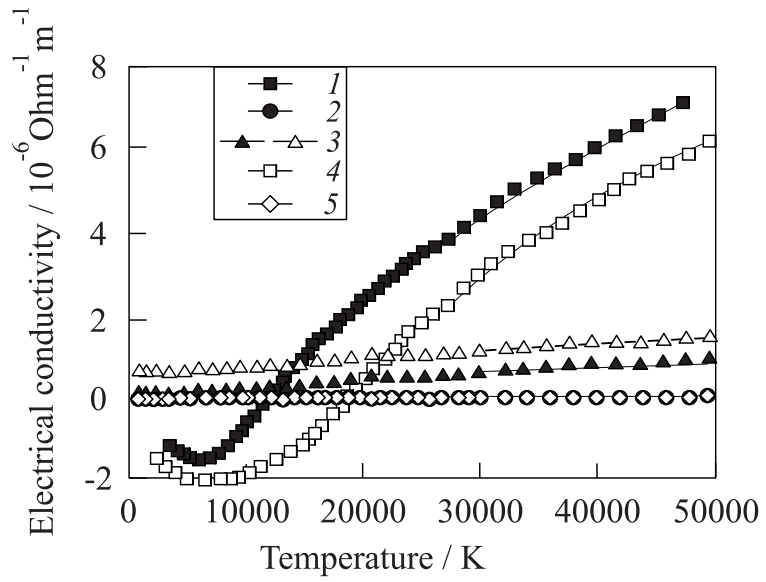

Figure 8 Electrical conductivity coefficient $\sigma_{c}^{d}$ as a function of $T$ for mixtures $\mathrm{N} / \mathrm{N}^{+} / e^{-}(0.98 / 0.01 / 0.01)$ (filled symbols) and $\mathrm{O} / \mathrm{O}^{+} / e^{-}(0.98 / 0.01 / 0.01)$ (empty symbols: $1-\sigma_{\mathrm{N}^{+} e^{-}} ; 2-\sigma_{\mathrm{N}^{+} \mathrm{N}^{+}} ; 3-\sigma_{e^{-} e^{-}} ; 4-\sigma_{\mathrm{O}^{+} e^{-}} ;$and $5-\sigma_{\mathrm{O}^{+} \mathrm{O}^{+}}$

With the temperature rise, the influence of electronic states on the thermal conductivity coefficient increases, and in the temperature ranges $T=5000$ $11,000 \mathrm{~K}$ for nitrogen and $T=7000-13,000 \mathrm{~K}$ for oxygen, neglecting electronic excitation may give an error up to $15 \%$. Finally, for higher temperatures, the internal (electronic) thermal conductivity may be three times larger than that for the translational degrees of freedom, which means that the electronic excitation gives the main contribution to the thermal conductivity coefficient.

It should be noted that in the present study, the heat transfer due to radiation is not considered. It is evident that for high temperatures, the radiative heat flux is of great importance, and it should be taken into account in the energy equation for the correct prediction of the flow parameters and total energy flux. However, the radiation does not influence the transport coefficients and, therefore, the main conclusions of this study are not affected by including or neglecting the radiative heat transfer. Moreover, as it is shown in [13], the heat transfer in chemically nonequilibrium flows is much less than that provided by the Planck function.

\section{CONCLUDING REMARKS}

A theoretical model for the transport properties in mixtures of ionized nitrogen $\left(\mathrm{N}, \mathrm{N}^{+}, e^{-}\right)$and oxygen $\left(\mathrm{O}, \mathrm{O}^{+}, e^{-}\right)$with electronic excitation of atoms is developed. On the basis of the proposed model, the heat conductivity, electri- 
cal conductivity, diffusion, thermal diffusion, shear viscosity, and bulk viscosity coefficients are calculated in the temperature range 500-50,000 K. The effect of electronic excitation on the transport properties is evaluated. It is shown that for $T>10,000 \mathrm{~K}$, accounting for electronic excitation becomes necessary for the correct calculation of the thermal conductivity coefficients calculation. Significant contribution of electrons to thermal conductivity in the temperature range $T=30,000-50,000 \mathrm{~K}$ is emphasized.

The results of this paper may improve the accuracy of the modeling for the spacecraft reentry.

Further improvement of the model requires taking into account electronic excitation of ions. Moreover, including neutral and ionized molecular species is necessary to improve the model.

\section{ACKNOWLEDGMENTS}

The authors are grateful to the Russian Foundation for Basic Research (Grant 11-01-00408) for the support of this study.

\section{REFERENCES}

1. Mason, E., and L. Monchick. 1962. Transport properties of polar gas mixture. J. Chem. Phys. 36:2746.

2. Ferziger, J., and G. Kaper. 1972. Mathematical theory of transport processes in gases. North-Holland, Amsterdam, London.

3. Ern, A., and V. Giovangigli. 1994. Multicomponent transport algorithms. SpringerVerlag, Lect. Notes Phys. Series monographs. M24.

4. Nagnibeda, E. A., and E. V. Kustova. 2009. Non-equilibrium reacting gas flows kinetic theory of transport and relaxation processes. Berlin-Heidelberg: SpringerVerlag.

5. Capitelli, M., G. Colonna, D. Giordano, L. Marraffa, A. Casavola, P. Minelli, D. Pagano, L. Pietanza, and F. Taccogna. 2005. Tables of internal partition functions and thermodynamic properties of high-temperature Mars-atmosphere scpecies from $50 \mathrm{~K}$ to $50000 \mathrm{~K}$. ESA STR-246. Noordwijk, The Netherlands: ESA Publications Division, ESTEC.

6. Bruno, D., A. Laricchiuta, M. Capitelli, and C. Catalfamo. 2007. Effect of electronic excited states on transport in magnetized hydrogen plasma. Phys. Plasmas. 14:022303.

7. Bruno, D., M. Capitelli, C. Catalfamo, and A. Laricchiuta,. 2007. Effect of electronic states on transport in magnetized hydrogen plasma. Phys. Plasmas. 14:072308. 
8. Kustova, E. V., and L. A. Puzyreva. 2009. Transport coefficients in non-equilibrium gas mixture flows with electronic excitation. Phys. Rev. E 80:046407.

9. Istomin, V.A., E. V. Kustova, and L. A. Puzyreva. 2011. Transport properties of electronically excited $\mathrm{N}_{2} / \mathrm{N}$ and $\mathrm{O}_{2} / \mathrm{O}$ mixtures. AIP Conference Proceedings, Rarefied Gas Dynamics. 1333:667-72.

10. Bruno, D., M. Capitelli, C. Catalfamo, R. Celiberto, G. Colonna, P. Diomede, D. Giordano, C. Gorse, A. Laricchiuta, S. Longo, D. Pagano, and F. Pirani. 2008. Transport properties of high-temperature mars-atmosphere components. Noordwijk, The Netherlands: ESA STR-256. ESA Publications Division, ESTEC.

11. Cenian, A., A. Chernukho, and V. Borodin. 1995. Modeling of plasma-chemical reactions in gas mixture of $\mathrm{CO}_{2}$ lasers. Contrib. Plasma Phys. 35:273-96.

12. Capitelli, M., C. Ferreira, B. Gordiets, and A. Osipov. 2005. Plasma kinetics in atmospheric gases. Berlin: Springer-Verlag.

13. Aliat, A., A. Chikhaoui, and E. V. Kustova. 2003. Nonequilibrium kinetics of radiative CO flow behind a shock wave. Phys. Rev. E 68:056306. 